\title{
Estructura interna de una escala de indefensión para jóvenes del norte de México
}

\author{
Internal Structure of a Scale of Helplessness for \\ young people in northern Mexico
}

\author{
José González-Tovar ${ }^{1}$ \\ Rosa Isabel Garza-Sánchez ${ }^{2}$ \\ Alicia Hernández-Montaño ${ }^{3}$ \\ ${ }^{1,3}$ Universidad Autónoma de Coahuila, Facultad de Psicología, México \\ ${ }^{2}$ Universidad Autónoma de Coahuila, Facultad de Trabajo Social, México
}

Resumen. Objetivo. Analizar la estructura interna de la propuesta de una Escala de Indefensión para Jóvenes (EIJ) en una muestra de la ciudad de Saltillo, Coahuila, México. Método. Se utilizó una metodología cuantitativa de diseño instrumental. Se eligió una muestra incidental de 750 casos, $50.3 \%$ hombres y $49.7 \%$ mujeres. La edad promedio fue de 21.85. Se utilizó la EIJ, que constó de 47 ítems que miden: afectividad, motivación, cognición y conducta. La confiabilidad fue de .898. Resultados. Se realizó el Análisis Factorial Exploratorio y Confirmatorio. Los índices de ajuste cumplieron con el criterio de buen ajuste. Esta herramienta aporta una forma de medir las variables predictoras de la conducta suicida, las cuales constituyen un problema de salud pública y que, en parte, son predichas por los indicadores de indefensión.

Palabras clave. Indefensión, jóvenes, análisis factorial.

Abstract. Objective. Analyze the internal structure of the proposal for a Youth Helplessness Scale (EIJ, by its acronym in Spanish) in a sample of the city of Saltillo, Coahuila, Mexico. Method. A quantitative methodology of instrumental design was used. An incidental sample of 750 cases was chosen, of which $50.3 \%$ were men and $49.7 \%$ women, and whose average age was 21.85. A Scale of Hopelessness for Young people consisting of 47 reactants was used, measuring affection, motivation, cognition and behavior. The reliability was. 898 , and an exploratory and confirmatory factor analysis was carried out. Results. Adjustment indices met the criterion of good fit. This tool provides a way to measure the predictor variables of suicidal behavior, all of which constitute a current public health problem, and which are partly predicted by indicators of helplessness.

Keywords. Hopelessness, youth, factor analysis.

${ }^{1} J o s e ́$ González-Tovar. Universidad Autónoma de Coahuila, Facultad de Psicología, México. Dirección Postal: Unidad Campo Redondo sin número, Edificio, Saltillo, Coahuila, México, Código Postal. 25280. E-mail: josegonzaleztovar@uadec.edu.mx

${ }^{2}$ Rosa Isabel Garza-Sánchez. Universidad Autónoma de Coahuila, Facultad de Trabajo Social, México. E-mail: isabelgarza@uadec.edu.mx

${ }^{3}$ Alicia Hernández-Montaño. Universidad Autónoma de Coahuila, Facultad de Psicología, México. E-mail: ahm123@hotmail.com

\section{@() $\Theta \Theta$}

Esta obra está bajo una licencia de Creative Commons Reconocimiento-NoComercial-SinObraDerivada 4.0 Internacional. 


\section{Introducción}

La desesperanza o indefensión se puede definir y explicar como un estado psicológico que se expresa mediante el pesimismo, así como la expectativa de que los resultados negativos son inevitables y la de incapacidad para cambiar ese destino o futuro (Abramson, Metalsky, \& Alloy 1989; Beck, Epstein, Brown, \& Steer, 1988; Ongen, 2012). De la misma manera, se ha vinculado este concepto con el alcoholismo, la sociopatía y la enfermedad física (Beck, Weissman, Lester, \& Trexler, 1974; Fanaj, Melonashi, \& Shkëmbi, 2015).

Entre las múltiples relaciones de este fenómeno, destacan aquellas que lo consideran como un potencial predictor tanto de la conducta, consumada y no consumada, como de la ideación suicida (Beck, 1986; Beck, Brown, Berchick, Stewart, \& Steer, 1990; Beck, Kovacs, \& Weissman, 1975; Boffa, King, Turecki, \& Schmidt, 2018; Liu et al., 2018; Rodríguez, Salvador, \& García-Alandete, 2017; Qiu, Klonsky, \& Klein, 2017).

La citada correlación con la conducta e ideación suicida hace necesario desarrollar métodos de evaluación de los rasgos de indefensión, ya que disminuirlos se relaciona con una baja en la propensión al suicidio (Cuijpers et al., 2013; Alsalman \& Alansari, 2016). A la par, mantiene una relación importante con la sensibilidad ante la ansiedad (Hudson, Wekerle, \& Stewart, 2015; Loxton, Bunker, Dingle, \& Wong, 2015; Castellanos-Ryan \& Conrod, 2012; Baines, Jones, \& Christiansen, 2016).

En otro sentido, también se ha relacionado a la indefensión con la calidad de vida relacionada con la salud, debido a que se ha encontrado que es un predictor de la mortalidad después de una enfermedad crítica, con la percepción de funcionalidad y con calidad de vida (Orwelius, Kristenson, Fredrikson, Walther, \& Sjöberg, 2017; Karakurt, Aşılar, Yildirim, \& Memiş, 2017).

Estudios recientes sobre universitarios con síntomas de depresión, de moderada a severa, han determinado que hay un efecto mediador significativo de los grupos de síntomas depresivos en la relación entre la indefensión y la ideación suicida (Tong, 2015); la indefensión de expectativas positivas en adolescentes de alto riesgo fue el único factor predictor significativo de síntomas depresivos y comportamiento suicida (Horwitz, Berona, Czyz, Yeguez, \& King, 2017).

La evaluación de la indefensión en los jóvenes es escasa, los estudios en esta población han asociado los niveles de indefensión con grados más elevados cuando se da alguna de estas variables: haber sido intimidados, haber sido asaltados por compañeros, haber tenido sexo forzado, ser parte de una pandilla, consumir alcohol de forma excesiva, haber hecho planes para intentar suicidarse e intentarlo (James, Reddy, Ellahebokus, Sewpaul, $\&$ Naidoo, 2017). Otros estudios han encontrado que los jóvenes consideran que el éxito no es alcanzable y, si no se tiene certeza, es más probable que experimenten emociones negativas como la ansiedad o indefensión (Burić \& Sorić, 2012).

Las características de personalidad de los jóvenes estudiantes tienen un papel determinante en la esperanza, decepción o indefensión. Mientras que una personalidad más amable y extrovertida aumenta la esperanza, la conciencia y el neuroticismo tienen un efecto directo sobre la indefensión (Bayrami et al., 2012). Por el contrario, según las investigaciones, la 
autoestima, el locus de control interno y la extraversión son predictores importantes de la indefensión (Mutlu, Balbag, \& Cemrek, 2010). Estos eventos adversos de la vida pueden incrementar el nivel de estrés que experimentan algunos jóvenes, al considerarlos como situaciones abrumadoras, violentas y difíciles de sobrellevar, generando en ellos sentimientos de depresión e indefensión (Arenas-Landgrave, Lucio-Gómez, \& Forns, 2012).

En cuanto a la medición, la Escala de Desesperanza de Beck (HBS) es una de las herramientas más utilizadas para medir el nivel de este factor presente en una población. La medición del constructo indefensión se concibe como un sistema de esquemas cognitivos que tienen en común expectativas negativas acerca del futuro, sea este el inmediato o más lejano (Aliaga, Rodríguez, Ponce, Frisancho, \& Enríquez, 2006). La HBS en pacientes colombianos con suicidabilidad presenta unas dimensiones similares a la versión original, con adecuada confiabilidad y moderada validez, tanto concurrente como predictiva (Rueda-Jaimes et al., 2016).

Cuando la meta es percibida como imposible de lograr, lo que surge es un estado de indefensión. Así, las emociones positivas o negativas serían el resultado del pensamiento esperanzado o desesperanzado en relación con los objetivos personales. Sin embargo, la indefensión no debe ser entendida como el extremo opuesto de la esperanza sino como un concepto específico y diferenciado del mismo, que puede contribuir a su comprensión, pero no como oposición (Arnau, Martínez, Niño de Guzmán, Herth, \& Yoshiyuki Konishi, 2010; Martínez Uribe, Cassaretto Bardales, \& Herth, 2012).

En México son pocos los estudios encontrados en donde se trabaje la ideación suicida y la indefensión. En una investigación con jóvenes de Coahuila de 15 a 29 años, se concluyó la presencia de riesgo en lo que respecta a la pérdida de motivación y expectativas negativas sobre el futuro, siendo estos predictores persistentes de la indefensión y el riesgo suicida (González \& Hernández, 2012). Otro estudio con estudiantes de preparatoria demostró que más de la mitad de los entrevistados tenían riesgo de indefensión cuando: experimentaban situaciones que trastornaban su vida, había ausencia de familiares en caso de problemas, haber sido víctimas de situaciones humillantes y existencia de insuficiencia económica (Córdova \& Rosales, 2010). En otro estudio, con 280 estudiantes de bachillerato, se encontró que el 5.7\% manifestó ideación suicida; al comparar con aquellos alumnos que no presentaron ideación suicida, se encontraron diferencias significativas en los factores de emoción negativa, comodidad, impulsividad, futuro negativo, futuro positivo, apoyo familiar y apoyo de amigos (Córdova, Rosales, Guzmán, \& Zúñiga, 2013).

Otras investigaciones se han enfocado en validar la consistencia interna y la estructura factorial de la Escala de Desesperanza de Beck en estudiantes mexicanos (Córdova \& Rosales, 2011); la ideación suicida y variables asociadas esto conforme al sexo (Rosales, Córdova, \& Guerrero, 2013); y la evaluación de la depresión, la ansiedad, la indefensión y el riesgo suicida en pacientes hospitalizados en México (Alderete-Aguilar, Cruz-Maycott, Candela-Iglesias, Rodríguez-Estrada, \& Reyes-Terán, 2017).

En México, los jóvenes representan uno de los grupos más vulnerables. La depresión, la ansiedad, la violencia, el consumo de sustancias adictivas y los problemas económicos y familiares potencian la tragedia. En la actualidad, el suicido y la depresión constituyen 
un problema de salud pública importante. En el 2015, según la Encuesta Nacional de los Hogares (ENG) realizada por el Instituto Nacional de Estadística y Geografía de México (INEGI, 2015), el 29.9\% de la población de 12 años o más manifestó sentirse deprimida alguna vez en su vida y el $65.5 \%$ de la población ha referido sentimientos de depresión algunas veces al año. En 2016, se registró un total de 6370 defunciones por suicidio en México (INEGI, 2018).

Las relaciones y los modelos construidos desde la experiencia empírica del trabajo con la indefensión requieren mayor profundidad y una consideración cultural para su medición, que permita apreciar adecuadamente su contexto y que aporte nuevas herramientas para su exploración. Esto con la finalidad de que, poco a poco, se consoliden como una alternativa para el diagnóstico. Por ello, la presente investigación tiene el objetivo de analizar la estructura interna de la primera versión de una Escala de Indefensión para Jóvenes en el contexto mexicano.

\section{Método}

Se utilizó un enfoque cuantitativo, con un diseño instrumental. De esta manera, los resultados permiten la comprobación de hipótesis que confirmen la estructura del instrumento propuesto (Ato, López, \& Benavente, 2013).

\section{Participantes}

Se eligió una muestra incidental de 750 casos. El 50.3\% correspondió a hombres y el $49.7 \%$ a mujeres, la muestra por sexo es equivalente $\left(\chi^{2}=.021, p=.884\right)$. La edad promedio fue de 21.85 años, con $D T=4.09$. La edad mínima fue de 15 años y la máxima de 29 . El 32\% se ubica en el rango de edad de 15 a 19 años; 34.5\% corresponde al grupo de 20 a 24 y el 33.5\% tiene entre 25 y 29 años. La proporción de casos por grupo quinquenal de edad es equitativo $\left(\chi^{2}=.728, p \leq .695\right)$. El $8.3 \%$ de la muestra no estudia ni trabaja; el $68.7 \%$ trabaja o estudia y el $23.1 \%$ realiza las dos actividades. La proporción de casos en cada grupo es diferente de manera significativa $\left(\chi^{2}=445.992, p \leq .001\right)$.

\section{Instrumentos}

Se utilizó un instrumento diseñado exprofeso para esta investigación, denominado Escala de Indefensión para Jóvenes (EIJ). Constó de 47 ítems agrupados en cuatro categorías conceptuales y apegadas al modelo de cuatro dimensiones de la indefensión de Alloy y Seligman (1979). De ahí se operacionalizaron los indicadores para ser agrupados en: afectivo (18 ítems), motivación (13 ítems), cognitivo (12 ítems) y conductual (4 ítems). Todas las dimensiones se responden con cinco opciones que van de $0=$ "nunca lo pienso, hago o siento" a 4 = "todo el tiempo lo pienso, hago o siento". Inicialmente, se calculó el coeficiente alfa de Cronbach para evaluar la confiabilidad para cada dimensión con la distribución y número de reactivos inicial, además de los intervalos de confianza para cada coeficiente, apegados a la recomendación de Domínguez-Lara y Merino (2015) y el procedimiento de Bonet (2002): afectivo $\alpha=.848$ (IC95\% .827-.867) motivación $\alpha$ $=.649$ (IC95\% .606-689), cognitivo $\alpha=.865$ (IC95\% .846-.882), conductual $\alpha=.652$ (IC95\% .609-.691). 


\section{Procedimiento y análisis de datos}

Los datos se recolectaron mediante el formato de auto reporte no remunerado. Los escenarios donde se llevaron a cabo las aplicaciones fueron los hogares e instituciones educativas a las que pertenecen los participantes del estudio. Se utilizó un consentimiento informado que garantiza la confidencialidad de la información. Además, la respuesta fue anónima y en todo momento se respetó el derecho de la persona participante a retirarse del estudio. Se exploró, en primera instancia, la distribución de los datos mediante la estadística descriptiva

Se realizó el Análisis Factorial Exploratorio (AFE) y el Análisis Factorial Confirmatorio (AFC). La muestra total se dividió en 50\% $(n=375)$ para el AFE y $50 \%$ para el AFC, para evitar validar la estructura sobre los mismos datos (Pérez-Gil, Chacón Moscoso, \& Moreno Rodríguez, 2000 ). Se exploró la adecuación de la matriz de correlaciones mediante la prueba de adecuación muestral de Káiser-Meyer-Olkin (KMO), valores cercanos a .90 indican una buena adecuación (Palacios, 2015). El método utilizado para la extracción de factores fue el Mínimos Cuadrados No Ponderados (ULS); este ha demostrado ser eficaz para la factorización de datos con variables ordinales y en casos de incumplimiento de la normalidad multivariada (Morata-Ramírez, Holado-Tello, Barbero-García, \& Méndez, 2015).

Para la extracción de los factores se utilizaron, además, tres criterios. El primero fue el criterio de autovalores mayores o iguales a uno; el segundo, fue el del punto de inflexión de la curva de Cattell (Lloret-Segura, Ferreres-Traver, Hernández-Baeza, \& Tomás-Marco, 2014); y, el tercero, fue el Análisis Paralelo (AP) con implementación optimizada (Lorenzo-Seva, Timmerman, \& Kiers, 2011). Se utilizó la matriz de correlaciones policóricas. Para la selección de los ítems se utiizó un carga factorial mínima de .30 (McDonald, 2014). Los análisis fueron realizados en los paquetes estadísticos SPSS 24 y Factor 10 (Lorenzo-Seva \& Ferrando, 2007).

Para el AFC se utilizaron como índices de bondad de ajuste el Error Cuadrático Medio de Aproximación (RMSEA), cuyo valor debe ser de menor o igual a .05 (Kline, 2005); el Índice de Ajuste Comparado (CFI); el Índice de Ajuste No Normado de Tucker y Lewis (NNFI), cuyo valor se recomienda que sea cercano o superior a 90 (Lévy \& Varela, 2006). También, se usaron el Índice de Bondad de Ajuste de Jöreskorg (GFI) y el Índice de Bondad de Ajuste Corregido Jöreskorg (AGFI). En estos dos últimos, los valores cercanos o superiores a .90 son los que muestran un mejor ajuste (Hair, Anderson, Tatham, \& Black, 1998). Por último, se usó la Raíz del Residuo Estandarizado Medio (RMSR); si este indicador se acerca a 0, puede considerarse un ajuste casi perfecto (Byrne, 2001).

\section{Resultados}

En el AFC se obtuvo un valor de KMO = .934, (LI = .932, LS = .933), que se considera como muy buena adecuación. La prueba esfericidad de Bartlett rechazó la hipótesis nula de independencia entre las variables $\left(\chi^{2}=13635, p \leq .001\right)$. En el procedimiento se descartaron dos ítems de la versión original del instrumento, ya que presentaron valores en la asimetría y la curtosis $\geq 2$. Estos son considerados como no aceptables (George \& Mallery, 2003).

El AP recomendó la solución de cuatro factores. Para esta solución, el Índice de Simplicidad Factorial de Bentler presentó un valor de $\mathrm{S}=.807$ (LI $=.734$, LS $=.877)$. El Índice de 
Simplicidad de Extracción de Factores fue de LS = .388 (LI = .377, LS = .418) (Bentler, 1977; Lorenzo-Seva, 2003). Estos indicadores demostraron que la solución recomendada por el AP fue la más simple; el número de factores conincide con el número original de dimensiones.

La varianza total explicada para una solución de cuatro factores, de acuerdo con el AFE, fue de 47.47\%. La varianza real, de acuerdo con el AP, fue de 57.7\%. Para mejorar las cargas factoriales y la claridad en la interpretación, se utilizó una rotación oblicua Promin.

El primer factor, etiquetado como Incapacidad de Autodeterminación, se compone de cuatro indicadores que miden la percepción de control sobre su vida y toma de decisiones, así como la capacidad para cumplir las metas y objetivos de vida. Este factor explica el $29.21 \%$ de la varianza; el AP reportó una varianza real de 36.4\%, el autovalor fue de 13.72 . La fiabilidad obtenida en este componente fue .608 (IC95\%).

El segundo componente, llamado Cognición y pérdida de esperanza, se integró por nueve elementos que determinan el nivel en que se encuentran presentes pensamientos automáticos negativos o de indefensión ante escenarios poco alentadores y satifactorios. varianza explicada de este factor fue de $8.86 \%$, el autovalor reportado fue de 4.16 , el reportado por el AP fue de 10.6, la fiabilidad fue de .859 (IC95\% 843-.873)(ver tabla 1).

Tabla 1

Resumen de cargas factoriales para una solución de cuatro factores de la Escala de Desesperanza

\begin{tabular}{lcc}
\hline \multicolumn{1}{c}{ Variable } & 1 & 2 \\
\hline 1. He sentido que no tengo la misma motivación para hacer las cosas como & .482 \\
anteriormente las hacía & .603 \\
2. Tiendo a posponer o evitar compromisos y responsabilidades & .493 \\
3. Me he sentido incapaz de cumplir o hacer las tareas más simples en mi vida & .538 \\
4. He dejado de hacer cosas porque pienso que no valen la pena o no son placenteras & .538 \\
Alfa de Ordinal & .608 & .479 \\
5. Podría decir que he llegado a perder la esperanza de amar & .571 \\
6. Si me ocurriera algo malo, sé que no hay alguien que me ayudaría para superarlo & .613 \\
7. He pedido ayuda hasta para hacer actividades cotidianas sin necesitarlo & .762 \\
8. Pienso que en unos años más la muerte será mejor opción en lugar de afrontar & \\
los problemas en mi vida & \\
9. En los últimos días, pienso que todo lo que hago o intento hacer me parece difícil & \\
10. Pienso que todo lo malo y lo bueno en mi vida se lo debo al destino & .500 \\
11. He perdido la esperanza de alcanzar mis sueños, metas, deseos, anhelos, etc. & \\
12. Pienso que es lo mismo el estar vivo o muerto & .457 \\
13. He llegado a pensar que las causas de todos mis problemas no están en mi \\
poder controlarlas
\end{tabular}

Nota. 1 = Incapacidad de Autodeterminación, 2= Cognición y perdida de esperanza. 
El tercer componente, etiquetado como Afectividad, mide el efecto de las emociones negativas consecuencia de la sensación de pérdida de control sobre su vida y la percepción de incapacidad. Este componente tiene una varianza explicada de 5.01\%, con un autovalor de 2.35. El valor real de la varianza, según el AP, fue de 5.7\%. El número de reactivos que integran este componente fue de 11 . El valor alfa de Cronbach fue de 822 (IC95\% .802-.840).

La Motivación fue el cuarto componente integrado. Incluye ocho ítems que determinan el impacto de un estado de indefensión en la motivación del sujeto para cumplir sus objetivos y cambiar su sentido de vida. Obtuvo una varianza explicada de $4.38 \%$. El Autovalor fue de 2.060; el AP reportó un valor real de la varianza de 5\%. La confiabilidad ordinal fue de .827 (IC95\% .807-.845)(ver Tabla 2).

Tabla 2

Resumen de cargas factoriales para una solución de cuatro factores de la Escala de Desesperanza (continuación)

14. Muy seguido me siento triste y me gana el llanto .570

15. Cuando tengo problemas con alguien a quien estimo, la tristeza aparece porque pienso que las cosas no mejorarán

16. He sentido tristeza y siento que no depende de mí dejar de sentirme así

17. Me he sentido triste más tiempo del que se diría que es "normal"

18. Tiendo a sentir enojo rápidamente por cosas sin importancia $\quad .516$

19. Busco pensar en cosas que me ayuden a olvidar los momentos de tristeza $\quad .630$

20. En ocasiones, he llegado a considerarme una persona infeliz $\quad .452$

21. Tiendo a usar el humor para que los sentimientos de tristeza sean menores $\quad .690$

22. Cuando siento tristeza o infelicidad, me ayuda platicar con otros para sentirme mejor $\quad .538$

23. He llegado a llorar sin motivo aparente o con mayor facilidad de lo normal $\quad .553$

24. En momentos de tristeza, he llegado a pensar que he hecho las cosas mal en $\quad .714$ mi vida

Alfa ordinal

25. Tengo motivos para luchar para vivir una vida larga y plena

26. La mayoría de los días son agradables y pasa rápido el tiempo

27. Me preocupa mi salud y siempre estoy al pendiente de ella

28. Disfruto mi vida y hacer las cosas que me gusta $\quad .656$

29. Me gustaría vivir toda mi vida tal y como la vivo ahora $\quad .696$

30. Actualmente, todo lo que me propongo lo consigo $\quad .784$

31. Siempre termino lo que comienzo $\quad .658$

32. Pienso que aún me quedan muchas cosas por hacer en mi vida 469

Alfa ordinal

Nota. 3 = Afectividad, $4=$ Motivación. 
Los valores de las correlaciones entre los factores indicaron valores que van de .156 para la asociación entre Motivación y Afectividad, hasta .664 para el valor más alto entre los factores Incapacidad de autodeterminación y Afectividad (ver Tabla 3).

El análisis confirmatorio (AC) se realizó con siete índices de ajuste, se obtuvieron intervalos de confianza al 95\% para cada uno. El valor del error cuadrático medio de aproximación (RMSEA) sugiere un ajuste adecuado, con un valor obtenido inferior a .060 (Anastasiadou \& Douma, 2014). El valor del Índice de Ajuste Comparado (CFI) y el Índice de Ajuste No Normado de Tucker y Lewis resultaron con valores superiores a .90, que representa buen ajuste (Novikova, Richman, Supekar, Barnard-Brak, \& Hall, 2013). Los índices de ajuste de Jöreskorg cumplieron con el criterio de valores superiores a .90. Además, la raíz del residuo estandarizado medio fue inferior a .080, recomendado como buen ajuste del modelo (Miranda-Zapata, Riquelme-Mella, Cifuentes-Cid, \& Riquelme-Bravo, 2014) (ver Tabla 4).

Tabla 3

Inter-correlaciones entre los factores obtenidos en el AFE

\begin{tabular}{lcccc}
\hline \multicolumn{1}{c}{ Factor } & 1 & 2 & 3 & 4 \\
\hline 1. Incapacidad de Autodeterminación & --- & --- & --- & --- \\
2. Cognición y pérdida de esperanza & .510 & --- & --- & --- \\
3. Afectividad & .664 & .524 & --- & --- \\
4. Motivación & -.205 & -.456 & -.156 & --- \\
\hline
\end{tabular}

Tabla 4

Índices de bondad de ajuste para la Escala de Desesperanza

\begin{tabular}{cccc}
\hline Indice & Valor & \multicolumn{2}{c}{ Intervalos de confianza al 95\% } \\
\cline { 3 - 4 } & & Límite inferior & Límite superior \\
\hline RMSEA & .025 & .025 & .025 \\
NNFI & .993 & .992 & .992 \\
CFI & .994 & .993 & .994 \\
GFI & .985 & .986 & .986 \\
AGFI & .982 & .983 & .983 \\
\hline
\end{tabular}

Nota. RMSEA = error cuadrático medio de aproximación, CFI = Índice de Ajuste Comparado, NNFI = Índice de Ajuste No Normado de Tucker y Lewis, GFI = Índice de Bondad de Ajuste de Joreskorg, AGFI = Índice de Bondad de Ajuste Corregido de Joreskorg, RMSR= Raíz del Residuo Estandarizado Medio. 
Se obtuvieron estadísticos descriptivos para cada una de las subescalas o dimensiones del instrumento, así como para la puntuación total obtenida. El procedimiento de cálculo de las puntuaciones se realizó mediante una suma simple. Mientras más alto sea el puntaje obtenido, mayor presencia de indicadores de indefensión en el respondiente. Cabe recordar que, si se revisan los ítems de la subescala de Motivación, las puntuaciones son inversas.

El promedio de las puntaciones de cada componente, incluida la calificación total de indefensión, se ubica en el percentil 50, en un punto medio entre el valor mínimo y máximo posible para cada aparado. No se encontró evidencia de sesgo importante, el perfil de la curtosis en todos los componentes es similar al mesocúrtico (ver Tabla 5).

Tabla 5

Estadísticos descriptivos para la versión final de la Escala de Desesperanza

\begin{tabular}{lcccccccccc}
\hline \multicolumn{1}{c}{ Puntuación } & Mín. & Máx & $M$ & $D T$ & As & $\mathrm{K}$ & \multicolumn{4}{c}{ Percentiles } \\
\cline { 6 - 10 } & & & & & & & 25 & 50 & 75 \\
\hline $\begin{array}{l}\text { Incapacidad de } \\
\text { Autodeterminación }\end{array}$ & .00 & 16 & 6.51 & 3.344 & .243 & -.210 & 4 & 6 & 9 \\
$\begin{array}{l}\text { Cognición y pérdida } \\
\text { de esperanza }\end{array}$ & .00 & 35 & 9.22 & 6.928 & .895 & .290 & 4 & 8 & 13 \\
Afectivo & 1 & 40 & 18.08 & 7.699 & .270 & -.215 & 13 & 18 & 23 \\
Motivación & .00 & 32 & 10.61 & 5.731 & .521 & .103 & 6 & 10 & 14 \\
Desesperanza & 5 & 117 & 44.43 & 18.14 & .490 & -.037 & 31 & 42 & 57 \\
\hline
\end{tabular}

Nota. As = coeficiente de asimetría, $\mathrm{K}=$ curtosis.

\section{Discusión}

La EIJ administrada en una muestra del norte de México reporta indicadores de confiabilidad adecuados, lo que implica una buena coherencia interna. Los factores demostraron correlación entre sí con valores de nivel medio. Lo anterior demuestra que esta versión inicial puede reportar datos consistentes en mediciones que se hagan en poblaciones con características similares. Además, el instrumento ajustó con una estructura de cuatro factores que es congruente con el modelo teorico de la indefensión (Alloy \& Seligman, 1979).

La contribución de esta herramienta aportará otra forma de medir la problemática de la ideación y la conducta suicida, las cuales son un actual problema de salud pública (Taskesen, Kanat, Cigir, \& Gor, 2012) y, en parte, son predichas por la presencia de indicadores de indefensión (Beck, 1986; Beck, Brown, Berchick, Stewart, \& Steer, 1990; Beck, Kovacs, \& Weissman, 1975; Boffa, King, Turecki, \& Schmidt, 2018). Identificarlas que permitiría, en futuras intervenciones, lograr una reducción de los niveles e indicadores de indefensión y, por lo tanto, una disminución de la tendencia suicida (Cuijpers et al., 2013; Alsalman \& Alansari, 2016). 
En cuanto a los factores obtenidos en la estructura de diseño del instrumento, se integraron cuatro factores que miden la indefensión: la incapacidad de autodeterminación, la motivación, la afectividad y las cogniciones asociadas a los estados de indefensión. En sucesivas aplicaciones, estos componentes podrían explicar, junto con otros instrumentos que midan variables como la ansiedad, a la cual la indefensión es sensible (Hudson, Wekerle, \& Stewart, 2015; Loxton et al., 2015; Castellanos-Ryan \& Conrod, 2012; Baines, Jones, \& Christiansen, 2016), sintomatología depresiva (Alloy \& Clements, 1998; Rodríguez-Naranjo \& Caño, 2016), estrés (Boffa et al., 2018) y algunas otras conductas de riesgo como el alcoholismo, la sociopatía y la enfermedad física (Beck et al., 1974; Fanaj et al., 2015).

En estudios subsecuentes donde se evalúe, también, la calidad de vida de los jóvenes, los ítmes podrían tener una relación inversa en el estado funcional, en el aspecto social, principalmente, y en la autoevaluación del estado de salud (Karakurt et al., 2017). Además, cabe resaltar que la disminución de los niveles de indefensión proporciona estrategias de afrontamiento de problemáticas relacionadas con la salud (Hernández \& Landero, 2015) y su consecuente desmoralización ante la falta de cumplimiento de expectativas (Grassi et al., 2010). Es allí donde los factores obtenidos de motivación y autodeterminación jugarían un papel importante para intervenir.

La limitación del estudio radica en la ausencia de una variable criterio para correlacionar los reactivos y puntuaciones de la escala propuesta. Además, no se utilizó una estrategia de muestreo probabilístico que mejore la posibilidad de generalización de los resultados, por lo que la línea de investigación a seguir comprende el ánalisis de nuevas muestras y el ánalisis de los diferentes niveles de invarianza de la estructura factorial del instrumento.

\section{Referencias}

Abramson, L. Y., Metalsky, G. I., \& Alloy, L. B. (1989). Hopelessness depression: A theory-based subtype of depression. Psychological Review, 96(2), 358-372. doi: 10.1037/0033-295X.96.2.358

Alderete-Aguilar, C., Cruz-Maycott, R., Candela-Iglesias, M. C., Rodríguez-Estrada, E., \& Reyes-Terán, G. (2017). Assessment of depression, anxiety, hopelessness and suicidal risk in HIV+ inpatients. Salud Mental, 40(1), 23-27. doi: 10.17711/ SM.0185-3325.2017.004

Aliaga, J., Rodríguez, L., Ponce, C., Frisancho, A., \& Enriquez, J. (2006). Escala de Desesperanza de Bech (BHS): adaptación y características psicométricas. Revista de Investigacion en Psicología, 9(1), 69-79. doi: 10.15381/rinvp.v9i1.4029

Alloy, L. B. \& Clements, C. M. (1998). Hopelessness theory of depression: Tests of the symptom component. Cognitive Therapy and Research, 22(4), 303-335. doi: 10.1023/A:1018753028007

Alloy, L. B. \& Seligman, M. E. P. (1979). On the Cognitive Component of Learned Helplessness and Depression. In G. H. Bower (Ed.), Psychology of Learning and Motivation (Vol. 13, pp. 219-276). Academic Press. doi: 10.1016/S0079-7421(08)60084-5

Actualidades en Psicología, 33(127), julio-diciembre 2019, 81-95 
Alsalman, R. \& Alansari, B. (2016). Relationship of suicide ideation with depression and hopelessness. European Psychiatry, 33(Supplement), S597. doi: 10.1016/j. eurpsy.2016.01.2228

Anastasiadou, S. D., \& Douma, S. T. (2014). A Structural Equation Model of Factors Affecting Medicine Profession Burnout. Procedia - Social and Bebavioral Sciences, 159, 387-390. doi: 10.1016/j.sbspro.2014.12.393

Arenas-Landgrave, P., Lucio-Gómez, E., \& Forns, M. (2012). Indicadores diferenciales de personalidad frente al riesgo de suicidio en adolescentes. Revista Iberoamericana de Diagnóstico y Evaluación - e Avaliação Psicológica, 1(33), 51-74. Recupeado de http:/ / www.redalyc.org $/$ articulo.oa?id $=459645437004$

Arnau, R. C., Martínez, P., Niño de Guzmán, I., Herth, K., \& Yoshiyuki Konishi, C. (2010). A Spanish-Language Version of the Herth Hope Scale: Development and Psychometric Evaluation in a Peruvian Sample. Educational and Psychological Measurement, 70(5), 808-824. doi: 10.1177/0013164409355701

Ato, M., López, J. J., \& Benavente, A. (2013). Un sistema de clasificación de los diseños de investigación en psicología. Anales de Psicología, 29(3), 1038-1059. doi: 10.6018/ analesps.29.3.178511

Baines, L., Jones, A., \& Christiansen, P. (2016). Hopelessness and alcohol use: The mediating role of drinking motives and outcome expectancies. Addictive Behaviors Reports, 4(Supplement C), 65-69. doi: 10.1016/j.abrep.2016.11.001

Bayrami, M., Heshmati, R., Ghotbi, M., Ghoradel, J. A., Hojatipor, H., \& Moslemifar, M. (2012). Relationship Between Personality Dimensions and Hopelessness: A Study on College Students. Procedia - Social and Behavioral Sciences, 46(Supplement C), 848852. doi: $10.1016 /$ j.sbspro.2012.05.211

Beck, A. T. (1986). Hopelessness as a Predictor of Eventual Suicide. Annals of the New York Academy of Sciences, 487(1), 90-96. doi: 10.1111/j.1749-6632.1986. tb27888.x

Beck, A. T., Brown, G., Berchick, R. J., Stewart, B. L., \& Steer, R. A. (1990). Relationship between hopelessness and ultimate suicide: a replication with psychiatric outpatients. American Journal of Psychiatry, 147(2), 190-195. doi: 10.1176/ajp.147.2.190

Beck, A. T., Epstein, N., Brown, G., \& Steer, R. A. (1988). An Inventory for Measuring Clinical Anxiety: Psychometric Properties. Journal of Consulting and Clinical Psychology, 56(6), 893-897. doi: 10.1037/0022-006X.56.6.893

Beck, A. T., Kovacs, M., \& Weissman, A. (1975). Hopelessness and Suicidal Behavior: An Overview. JAMA: The Journal of the American Medical Association, 234(11), 1146-1149. doi: $10.1001 /$ jama.1975.03260240050026

Beck, A. T., Weissman, A., Lester, D., \& Trexler, L. (1974). The measurement of pessimism: The Hopelessness Scale. Journal of Consulting and Clinical Psychology, 42(6), 861-865. doi: $10.1037 / \mathrm{h} 0037562$ 
Bentler, P. M. (1977). Factor simplicity index and transformations. Psychometrika, 42(2), 277-295. doi: 10.1007/bf02294054

Boffa, J. W., King, S. L., Turecki, G., \& Schmidt, N. B. (2018). Investigating the role of hopelessness in the relationship between PTSD symptom change and suicidality. Journal of Affective Disorders, 225, 298-301. doi: 10.1016/j.jad.2017.08.004

Bonett, D. G. (2002). Sample Size Requirements for Testing and Estimating Coefficient Alpha. Journal of Educational and Behavioral Statisitcs, 27(4), 335-340. doi: $10.3102 / 10769986027004335$

Burić, I. \& Sorić, I. (2012). The role of test hope and hopelessness in self-regulated learning: Relations between volitional strategies, cognitive appraisals and academic achievement. Learning and Individual Differences, 22(4), 523-529. doi: 10.1016/j.lindif.2012.03.011

Byrne, B. M. (2001). Structural equation modeling with AMOS. Basic concepts, applications and programming. Mahwah, NJ: Lawrence Eribaum Associates.

Castellanos-Ryan, N. \& Conrod, P. (2012). Personality and substance misuse: Evidence for a four-factor model of vulnerability. In J. C. Verster, K. Brady, M. Galanter, \& P. Conrod (Eds.), Drug Abuse and Addiction in Medical Illness: Causes, Consequences and Treatment (pp. 47-62). New York, NY, US: Springer Science + Business Media. doi: 10.1007/978-1-4614-3375-0_4

Córdova , M., Rosales, J., Guzmán, B., \& Zúñiga, G. (2013). Características del estudiante con y sin ideación suicida del colegio de bachilleres de San Luis Potosí (México). Saludy Sociedad, 4(2), 120-134. doi: 10.22199/S07187475.2013.0002.00001

Córdova, M. \& Rosales, J. C. (2010). La desesperanza de riesgo en jovenes mexicanos y aspectos sociodemograficos asociados: diferencias por sexo. Psicología y salud, 20(2). doi: 10.25009/pys.v20i2.602

Córdova, M. \& Rosales, J. C. (2011). Consistencia interna y estructura factorial de la Escala de Desesperanza de Beck en estudiantes mexicanos. Revista de Psicologia, 29(2), 289-309. Recuperado de http://revistas.pucp.edu.pe/index.php/psicologia/ article/view/2470/2419

Cuijpers, P., de Beurs, D. P., van Spijker, B. A. J., Berking, M., Andersson, G., \& Kerkhof, A. J. F. M. (2013). The effects of psychotherapy for adult depression on suicidality and hopelessness: A systematic review and meta-analysis. Journal of Affective Disorders, 144(3), 183-190. doi: 10.1016/j.jad.2012.06.025

Domínguez-Lara, S. \& Merino, C. (2015). ¿Por qué es importante reportar los intervalos de confianza del coeficiente alfa de Cronbach? Revista Latinoamericana de Ciencias Sociales, niñezy Juventud, 13(2), 1326-1328. Recuperado de http:/ / revistaumanizales. cinde.org.co/rlcsnj/index.php/Revista-Latinoamericana/article/view/2030/629

Fanaj, N., Melonashi, E., \& Shkëmbi, F. (2015). Self-esteem and Hopelessness as Predictors of Emotional Difficulties: A Cross-sectional Study among Adolescents in Kosovo. Procedia - Social and Behavioral Sciences, 165 (Supplement C), 222-233. doi: 10.1016/j.sbspro.2014.12.626

Actualidades en Psicología, 33(127), julio-diciembre 2019, 81-95 
George, D. \& Mallery, M. (2003). Using SPSS for Windows Step by Step: a simple guide and reference. Boston, MA: Allyn \& Bacon.

González, J. \& Hernández, A. (2012). La desesperanza aprendida y sus predictaros en jovenes: Análisis desde el modelo de Beck. Enseñanza e Investigación en Psicología, 17(2), 313- 327. Recuperado de http://www.redalyc.org/articulo.oa?id=29224159015

Grassi, L., Travado, L., Gil, F., Sabato, S., Rossi, E., Tomamichel, M., . . . Nanni, M. G. (2010). Hopelessness and Related Variables Among Cancer Patients in the Southern European Psycho-Oncology Study (SEPOS). Psychosomatics, 51(3), 201207. doi: 10.1016/S0033-3182(10)70686-1

Hair, J., Anderson, R., Tatham, R., \& Black, W. (1998). Análisis Multivariante (5ª ed). Madrid, Spain: Prentice Hall.

Hernández Moreno, F. P. \& Landero Hernández, R. (2015). Aspectos Psicosociales Relacionados con el TEPT en Pacientes con Cáncer de Mama. Acta de Investigación Psicológica, 5(1), 1881-1891. doi: 10.1016/S2007-4719(15)30008-9

Horwitz, A. G., Berona, J., Czyz, E. K., Yeguez, C. E., \& King, C. A. (2017). Positive and Negative Expectations of Hopelessness as Longitudinal Predictors of Depression, Suicidal Ideation, and Suicidal Behavior in High-Risk Adolescents. Suicide and Life-Threatening Behavior, 47(2), 168-176. doi: 10.1111/sltb.12273

Hudson, A., Wekerle, C., \& Stewart, S. H. (2015). Associations between personality and drinking motives in adolescents involved in the child welfare system. Personality and Individual Differences, 81, 84-89. doi: 10.1016/j.paid.2014.12.008

Instituto Nacional de Estadística y Geografía de México. [INEG]. (2015). Encuesta Nacional de Hogares. Recuperado de http://www.beta.inegi.org.mx/app/ indicadores $/$ ?ind $=6200240546$

Instituto Nacional de Estadística y Geografía de México. [INEGI]. (2018). Suicidios registrados (Defunciones). Recuperado de http://www.beta.inegi.org.mx/app/ indicadores $/$ ? ind $=6200240338 \#$

James, S., Reddy, S. P., Ellahebokus, A., Sewpaul, R., \& Naidoo, P. (2017). The association between adolescent risk behaviours and feelings of sadness or hopelessness: a cross-sectional survey of South African secondary school learners. Psychology, Health and Medicine, 22(7), 778-789. doi: 10.1080/13548506.2017.1300669

Karakurt, P., Aşılar, R. H., Yildirim, A., \& Memiş, Ş. (2017). Determination of Hopelessness and Quality of Life in Patients with Heart Disease: An Example from Eastern Turkey. Journal of Religion and Health, 1-16. doi: 10.1007/s10943-017-0456-3

Kline, R. B. (2005). Principles and practice of structural equation modeling. New York, Estados Unidos: Guilford Press. Segunda Edición.

Lévy J. P. \& Varela J. (2006). Modelización con estructuras de covarianzas en ciencias sociales. Madrid: Netbiblo. 
Liu, Z. Z., Chen, H., Bo, Q. G., Chen, R. H., Li, F. W., Lv, L., . . Liu, X. (2018). Psychological and behavioral characteristics of suicide attempts and non-suicidal self-injury in Chinese adolescents. Journal of Affective Disorders, 226, 287-293. doi: 10.1016/j.jad.2017.10.010

Lloret-Segura, S., Ferreres-Traver, A., Hernández-Baeza, A., \& Tomás-Marco, I. (2014). El Análisis Factorial Exploratorio delos Ítems: una guía práctica, revisada y actualizada. Anales de Psicología, 30(3), 1151-1169. doi: 10.6018/analesps.30.3.199361

Lorenzo-Seva, U. (2003). A factor simplicity index. Psychometrika, 68(1), 49-60. doi: $10.1007 / \mathrm{bf} 02296652$

Lorenzo-Seva, U. \& Ferrando, P. (2007). FACTOR: A computer program to fit the exploratory factor analysis model. Behavior Research Methods, 38(1), 88-91. doi: 10.3758/BF03192753

Lorenzo-Seva, U., Timmerman, M. E., \& Kiers, H. A. (2011). The Hull Method for Selecting the Number of Common Factors. Multivariate Behav Res, 46(2), 340-364. doi: 10.1080/00273171.2011.564527

Loxton, N. J., Bunker, R. J., Dingle, G. A., \& Wong, V. (2015). Drinking not thinking: A prospective study of personality traits and drinking motives on alcohol consumption across the first year of university. Personality and Individual Differences, 79, 134-139. doi: 10.1016/j.paid.2015.02.010

Martínez Uribe, P., Cassaretto Bardales, M., \& Herth, K. (2012). Propiedades psicométricas de la Escala de Esperanza de Herth en español. Revista Iberoamericana de Diagnóstico y Evaluación - e Avaliação Psicológica, 1(33), 127-145. Recuperado de http://www. redalyc.org/articulo.oa?id $=459645437008$

McDonald, R. P. (2014). Factor analysis and related methods. New York: Psychology Press. doi: $10.4324 / 9781315802510$

Miranda-Zapata, E., Riquelme-Mella, E., Cifuentes-Cid, H., \& Riquelme-Bravo, P. (2014). Análisis factorial confirmatorio de la Escala de habilidades sociales en universitarios chilenos. Revista Latinoamericana de Psicología, 46(2), 73-82. doi: 10.1016/S0120-0534(14)70010-X

Morata-Ramírez, M. A., Holgado-Tello, F. P., Barbero-García, I., \& Méndez, G. (2015). Análisis factorial confirmatorio: recomendaciones sobre mínimos cuadrados no ponderados en función del error Tipo I de Ji-Cuadrado y RMSEA. Acción Psicológica, 12, 79-90. doi: 10.5944/ap.12.1.14362

Mutlu, T., Balbag, Z., \& Cemrek, F. (2010). The role of self-esteem, locus of control and big five personality traits in predicting hopelessness. Procedia - Social and Behavioral Sciences, 9(Supplement C), 1788-1792. doi: 10.1016/j.sbspro.2010.12.401

Novikova, S. I., Richman, D. M., Supekar, K., Barnard-Brak, L., \& Hall, D. (2013). Chapter Three - NDAR: A Model Federal System for Secondary Analysis in Developmental Disabilities Research. In R. C. Urbano (Ed.), International Review of Research in Developmental Disabilities (Vol. 45, pp. 123-153): Academic Press. doi: 10.1016/B978-0-12-407760-7.00003-7 
Ongen, D. E. (2012). The Relationship between Hopelessness and Sensation Seeking in Secondary School Teacher Candidates. Procedia - Social and Behavioral Sciences, 46(Supplement C), 3021-3025. doi: 10.1016/j.sbspro.2012.06.002

Orwelius, L., Kristenson, M., Fredrikson, M., Walther, S., \& Sjöberg, F. (2017). Hopelessness: Independent associations with health-related quality of life and short-term mortality after critical illness: A prospective, multicentre trial. Journal of Critical Care, 41, 58-63. doi: 10.1016/j.jcrc.2017.04.044

Palacios, J. R. (2015). Estimación psicométrica de la escala de autoeficacia ante conductas de riesgo para adolescentes en México. Psychosocial Intervention, 24(1), 1-7. doi: 10.1016/j.psi.2014.11.004

Pérez Gil, J.A., Chacón Moscoso, S. y Moreno Rodríguez, R. (2000). Validez de constructo: el uso de análisis factorial exploratorio-confirmatorio para obtener evidencias de validez. Psicothema, 12 (2), 442-446. Recuperado de: http:/ /www. psicothema.com/pdf/601.pdf

Qiu, T., Klonsky, E. D., \& Klein, D. N. (2017). Hopelessness Predicts Suicide Ideation But Not Attempts: A 10-Year Longitudinal Study. Suicide and Life-Threatening Behavior. doi: $10.1111 /$ sltb. 12328

Rodríguez, S. P., Salvador, J. H., \& García-Alandete, J. (2017). El papel de la desesperanza y el sentido de la vida en una muestra clínica con autolesiones no suicidas e intentos suicidas. Psicothema, 29(3), 323-328. doi: 10.7334/psicothema2016.284

Rodríguez-Naranjo, C., \& Caño, A. (2016). Daily stress and coping styles in adolescent hopelessness depression: Moderating effects of gender. Personality and Individual Differences, 97(Supplement C), 109-114. doi: 10.1016/j.paid.2016.03.027

Rosales, J. C., Córdova, M., \& Guerrero, D. I. (2013). Ideación suicida y variables asociadas, conforme al sexo, en estudiantes de la Universidad Tecnológica del Estado de Puebla. Revista Electrónica de Psicologia Iztacala, 16(2), 390-406. Recuperado de http://www. iztacala.unam.mx/carreras/psicologia/psiclin/vol16num2/Vol16No2Art5.pdf

Rueda-Jaimes, G. E., Castro-Rueda, V. A., Rangel-Martínez-Villalba, A. M., MorenoQuijano, C., Martinez-Salazar, G. A., \& Camacho, P. A. (2016). Validación de la Escala de Desesperanza de Beck en pacientes con riesgo suicida. Revista de Psiquiatría y Salud Mental. doi: 10.1016/j.rpsm.2016.09.004

Taskesen, O., Kanat, S., Cigir, E., \& Gor, T. K. (2012). A Study on Hopelessness Levels of High School Students According to Their Need They Feel About Receiving Art Education Informally. Procedia - Social and Behavioral Sciences, 51(Supplement C), 340-347. doi: 10.1016/j.sbspro.2012.08.170

Tong, E. M. W. (2015). Hope and Hopelessness. In J. D. Wright (Ed.), International Encyclopedia of the Social \& Behavioral Sciences (Second Edition) (pp. 197-201). Oxford: Elsevier. doi: 10.1016/B978-0-08-097086-8.25013-7 